\title{
Italique
}

Poésie italienne de la Renaissance

I| 1998

Varia

\section{Dante, l'Aviso piacevole et Henri de Navarre}

\section{Jean Balsamo}

\section{OpenEdition}

\section{Journals}

Édition électronique

URL : http://journals.openedition.org/italique/89

DOI : $10.4000 /$ italique.89

ISSN : 1663-4438

\section{Éditeur}

Librairie Droz

\section{Édition imprimée}

Date de publication : 1 juin 1998

Pagination : 79-94

ISBN : 2-600-00239-1

ISSN : 1423-3983

\section{Référence électronique}

Jean Balsamo, «Dante, l'Aviso piacevole et Henri de Navarre », Italique [En ligne], I | 1998, mis en ligne le 14 septembre 2009, consulté le 01 mai 2019. URL : http://journals.openedition.org/italique/89 ; DOI : 10.4000/italique.89 
JEAN BALSAMO

DANTE, L'AVISO PIACEVOLE

ET HENRI DE NAVARRE 


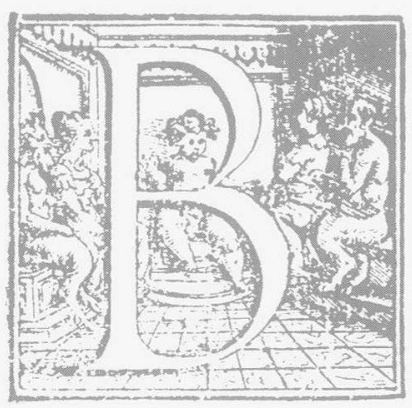

Althazar Grangier fut l'auteur de la première traduction française de la Commedia', publiée en 1596. Cette édition, dédiée au roi Henri IV par un de ses aumôniers, venait après la réconciliation du roi avec Rome et célébrait ses retrouvailles avec son royaume. Elle ne confirmait pas, au contraire, l'accueil et la reconnaissance de Dante par les lettres françaises. Grangier était conscient de la difficulté de l'ouvre du poète florentin, au "stile concis, difficile et particulier à un seul", comme de l'étrange illisibilité de sa propre traduction qui réclamait des "façons de parler anciennes, non communes". L'apologie de Dante ("un poète excellent, un philosophe profond et un théologien judicieux") qui ouvre la préface risque ainsi de fausser, par son caractère exceptionnel, linterprétation que l'on peut donner de la réception du poète toscan en France, à la fin de la Renaissance. La traduction de Grangier constituait un aboutissement plus quiun point de départ, le résultat de quelques initiatives isolées dont il convient de ne pas surestimer la portée et qu'Arturo Farinelli, dans un ouvrage fondateur, voyait plutôt comme la preuve paradoxale de l'infortune française de Dante ${ }^{2}$. Cette expression toutefois péchait aussi par excès. Pour le savant italien, qui approfondissait des suggestions offertes par Henri Hauvette et Emile Picot, les lettrés français du XVI siècle, séduits par les faux prestiges du pétrarquisme, auraient été incapables de comprendre le haut savoir d'un poète véritablement inspiré, peu compatible avec les futilités de l'art de cour. Cette thèse simposa comme une vérité pendant soixante ans, jusqu'à ce que Franco Simone fit apparaître que cette évidence critique répondait à un faux problème, mal résolu parce qu'il avait été mal posé ${ }^{3}$. Les Français avaient connu Dante, ou du moins son nom, avaient lu son poème et d'autres æuvres, l'avaient traduit, mais ils l'avaient interprété de manière sélective, en relation à d'autres auteurs, selon les termes de leur culture nationale et sans rendre un hommage particulier au poète. Véritable signum contradictionis de la culture des Valois, Dante, ce "poète fort ignorant ${ }^{4} »$, sur qui pesait toujours le soupçon d'un irréductible «misogallisme» excusé avec peine par son traducteur Grangier au prix de certains accommodements ("mais telles choses sont dictes par métafore"), avait eu, à défaut de séduire par ses vers, du moins le mérite d'offrir une contribution à la langue royale et des armes contre la cour de Rome.

Le rôle joué par Dante ne sestimera ni dans les éditions de son grand poème publiées ou reçues en France, ni dans la fréquence des citations que tel ou 
tel érudit faisait de ses autres textes. Les éditions lyonnaises de la Commedia, celle fort médiocre publiée par Jean de Tournes et adressée à Maurice Scève, ou les quatre autres données entre 1551 et 1575 avec beaucoup plus de soin typographique et philologique par Guillaume Rouillé aidé par Luc Antonio Ridolfi, étaient plutôt destinées au marché italien de Lyon et de la Péninsules. Les bibliothèques privées dont nous connaissons les inventaires n'abondaient pas en exemplaires du poème de Dante. Quelques rares amateurs en possédaient une édition, des poètes tels Belleau ${ }^{6}$ et Desportes, dont on connaît aussi l'exemplaire de l'Amoroso Convivio aujourd'hui dans une collection particulière, des gens de robe qui avaient connu l'Italie, les Robertet, les Du Prat, des curieux, tel Pierre de L'Estoile, attentif à trouver des analogies historiques pour comprendre la situation politique de son temps ${ }^{7}$. Nicolas Colin, le savant secrétaire des cardinaux de Lorraine et de Guise, avait réuni une belle collection de poètes italiens ; Dante n'y figurait que dans la traduction de Grangier classée parmi les livres français ${ }^{8}$. Le catalogue de la Bibliotheca Thuana de Jacques-Auguste de Thou, le plus fameux bibliophile de son siècle, recensait au contraire plusieurs éditions italiennes de la Commedia ; étaitce une preuve du brusque succès de cette cuvre sous le règne de Henri IV? Il faut en tout cas faire la part des livres offerts, du collectionnisme naissant, de l'attention accordée aux beaux livres de provenance prestigieuse : parmi les volumes de De Thou figurait en bonne place le Dante de Grolier. Un exemplaire de l'édition Sessa de 1564, revêtu d'une somptueuse reliure à la fanfare, appartenait au financier Moreau d'Auteuil': indiquait-il un goût particulier pour le poète italien, était-ce un précieux objet offert par un partisan d'origine florentine, un Gondi ou un Rucellai, pour qui Dante pouvait être plus quiun nom? Et que dire du Dante que possédait le cardinal de Bourbon ${ }^{10}$ ? C'était un exemplaire de l'édition publiée par Rouillé en 1571 comme le Dante de Catherine de Médicis, tous les deux probablement des exemplaires d'hommage plutôt que des exemplaires personnels ${ }^{11}$.

Les fragments de traduction, les références, les citations étaient à peine moins rares, et ces dernières pouvaient provenir de multiples médiations. Montaigne citait bien Dante, sans le nommer pourtant : un vers de l'Inferno qui illustrait sa critique de la présomption, "Non men che saper dubbiar maggrada» et trois vers très imagés du Purgatorio à valeur d'exemple ; il tirait ces vers de la Civil Conversazione de Guazzo et de l'Ercolano de Varchi ${ }^{12}$. Papire Masson alléguait Dante, "historicus magis quam poeta", comme une autorité parmi d'autres pour écrire l'histoire des papes et, en composant une Vita du poète qui servait comme de prologue 
aux biographies bien plus amples qu'il consacrait à Pétrarque et à Boccace, il citait en italien vingt-sept vers du Purgatorio, afin d'obtenir, disait-il, le même effet d'étrangeté que les citations grecques chez Cicéron ${ }^{13}$. A sa suite, Antoine Du Verdier reprit quelques vers du poète dans les notices de sa Prosopographie consacrées aux papes Sylvestre II et Nicolas IV ${ }^{14}$. Dans l'entourage de François d'Alençon, à la suggestion de Guillaume Postel, Jean de La Gessée et Guy Le Fèvre de La Boderie traduisirent quelques bref extraits ${ }^{15}$. Dante, en fait, restait le plus souvent un simple nom connu de façon indirecte, appelé par d'autres noms plus fameux, que venaient illustrer quelquefois des fragments d'une langue singulière et vieillie. Jean Lemaire de Belges, Pasquier, Jean de Nostredame, Fauchet citaient Dante ou le nommaient. Le nom du poète florentin faisait partie de l'histoire des lettres italiennes comme un nom emblématique, cétait la première des "trois couronnes de l'Italie", le premier des "tres Hetruriae proceres", l'ancêtre sinon le fondateur de la langue vulgaire. Cette histoire était celle qu'avaient transmise les érudits transalpins aux érudits français. Ceux-ci se bornaient à reprendre leurs formules dans le discours très cohérent qu'ils tenaient sur les lettres italiennes, toujours considérées dans une relation de subordination aux lettres françaises ${ }^{16}$. Dante était au mieux le premier en date, mais non pas le plus important des promoteurs du vulgaire ; l'italien naissait véritablement avec Pétrarque. Dans une élégie adressée à son ami le poète Bartolomeo Del Bene, Ronsard traçait nettement la ligne de partage entre la lumière et les ténèbres, entre la grande poésie et les balbutiements de la langue, entre Pétrarque et "la Nuit // De Dante et de Cavalcanti ${ }^{17}$ ". Dante était reçu en France à travers le filtre et le canon littéraire des Italiens, il était aussi soumis aux catégories d'une histoire littéraire française volontiers schématique. Il illustrait une étape, une solution étroitement "toscane» dans l'émancipation du vulgaire italien, qui était elle-même la préparation et l'annonce de l'émancipation de la langue française, langue royale, et de son essor. Le Fèvre de La Boderie évoquait le poète italien de façon significative dans sa Galliade. Il représentait Dante, sous la conduite de Virgile dont il acceptait le legs, donnant l'hospitalité aux Muses qui regagnaient leur véritable patrie, la France des Valois ${ }^{18}$. Enfin, les liens entre Dante et les poètes provençaux ne cessaient d'être soulignés, comme était mis en exergue le séjour que le poète exilé avait fait à Paris ; ils témoignaient assez, selon Nostredame et Pasquier, de tout ce que le grand-père des lettres italiennes devait à la culture française, dont eux-mêmes pouvaient ainsi revendiquer la primauté.

Il fallut toutes les arrière-pensées d'un fuoruscito florentin pour donner à Dante une brève notoriété. Jacopo Corbinelli édita à Paris le De Vulgari 
Eloquentia. L'ouvrage, publié en 1577, sinsérait entre deux autres textes qui rappelaient son enjeu politique, celui d'une "controcultura dell'esilio", selon l'heureuse expression de Paolo Simoncelli'. L'éloge de la langue curiale était éclairé par les Consigli e avvertimenti de Guichardin et par un texte moins connu, le De Principatu de Mario Salamonio degli Alberteschi, qui esquissait une théorie contractuelle du pouvoir. La publication de ces trois ouvrages sous le patronage de membres influents de la haute administration royale obéissait en premier lieu à une claire intention polémique, anti-médicéenne, et elle sinscrivait dans les illusions d'une restauration républicaine à Florence. Ce fut bien à Florence que le De Vulgari Eloquentia d'un Corbinelli qui se jugeait devenu malgré lui "barbaro e tutto francese" suscita un véritable débat. En publiant cet ouvrage contesté, Corbinelli entendait aussi prouver que Dante en était l'auteur, contre l'avis de Varchi et des Académiciens. En l'éditant pour la première fois dans son texte original, il voulait contester la solution étroitement florentine donnée par les érudits italiens au problème de la langue vulgaire et leur référence stérilisante aux "tre corone». L'exemple de Dante faisait apparaître la composante provençale, française en somme, du modèle florentin, et Corbinelli pouvait alors élargir à d'autres auteurs, Jacopone da Todi et Catherine de Sienne, le canon linguistique de l'italien moderne quill envisageait. Ce texte confidentiel qui reflétait les débats les plus pointilleux des milieux académiques florentins ne fut pas moins diffusé à la cour de France, dans un sens bien différent, comme un véritable panégyrique de la langue royale, au rebours même de l'intention anti-française qui l'animait à l'origine ${ }^{20}$. La composante curiale était confirmée par le réseau des pièces liminaires qui associaient pour un même hommage Baïf, Postel et le secrétaire d'Etat Pierre Forget, protecteur de Corbinelli et dédicataire de l'édition. Lu par quelques érudits français, parmi lesquels Papire Masson, qui le mentionnait dans sa Vita, et Pierre Tamisier, futur traducteur de Savonarole ${ }^{21}$, le petit volume édité par Corbinelli précisait la mince réputation de Dante pour les Français : moins celle d'un poète que d'un parmi les "nombreux autheurs modernes qui ont escrit de la langue italienne», à l'égal d'un Bembo, d'un Dolce ou d'un Speroni ${ }^{22}$.

Le nom de Dante était parfois invoqué dans le discours réticent que les érudits français tenaient sur les lettres italiennes. Il servit avec plus de force dans la polémique anti-romaine, point de rencontre politique des propagandes gallicane et protestante ${ }^{23}$. Au mois de septembre 1585, par la bulle $\mathrm{Ab}$ immensa xterni regis potentia, Sixte Quint excommuniait Henri de Condé et son cousin Henri de Navarre, qu'il excluait ipso facto 
de la succession au trône de France préparée par Henri III. Cette mesure suscita l'indignation du prétendant, du roi et du Parlement, et fit naître des libelles hostiles à l'ingérence pontificale dans les affaires de France. L'ambassadeur de Savoie, René de Lucinge, écrivit au duc pour expliquer le mystère d'une unanimité retrouvée entre catholiques et protestants : "ils tiennent icy que les roys de France sont exempts des censures de l'Eglise pour l'accord faict par Philippe le Bel avec le successeur de Boniface VIII duquel il avoit esté excommunié24". Lucinge annonçait la publication probable de "quelque livre sur ce faict» dont il allait se mettre en quête. Plusieurs des textes suscités par l'affaire sont connus. Pierre de L'Estoile rédigea en réponse à la bulle un pamphlet qui fut affiché à Rome contre la statue de Pasquin ${ }^{25}$. Pierre de Belloy, porte-parole du roi de Navarre, réfuta la décision pontificale dans son traité Moyens d'abus, entreprises et nullités du rescrit et bulle du Pape. François Hotman donna une réponse savante, le Brutum Fulmen. Ni Belloy ni Hotman ne citaient Dante. Hotman pourtant connaissait bien les épîtres Sine nomine de Pétrarque et les autres auteurs italiens qui avaient écrit contre la cour pontificale:

Comme si en celle ville qui est le repaire de toute sorte d'impurité, on ne contrevenoit point à l'ordonnance de Dieu : Petrarque toutefois, Mantuan, Sanazare, et plusieurs autres Italiens l'appellent boutique de toutes meschancetés, Babylone, Sodome, escole d'erreur, temple d'hérésie et putain effrontée ${ }^{26}$.

Un dernier texte sinscrivait dans cette polémique. Dante, ignoré des juristes ou seulement cité par allusion parmi les "autres Italiens", prenait un relief singulier. En 1586, en effet, parut sous la fausse adresse ironiquement monacale de "Monaco appresso Giovanni Swartz", un Aviso piacevole poétique donné à l'Italie par un soi-disant gentilhomme français; celui-ci faisait précéder une belle collection de sonnets anti-romains d'une anthologie tirée des trois pères des lettres italiennes, et donnait dixneuf extraits du grand poème de Dante avec leur commentaire ${ }^{27}$. On a longtemps cru que l'ouvrage avait été imprimé en Hollande ou à Genève, on a même pu suggérer Montauban ou La Rochelle. L'ouvrage provenait en fait des presses londoniennes de John Wolfe ${ }^{28}$. Cet imprimeur, protégé par Philip Sidney, fut un des plus actifs promoteurs de l'italianisme élisabéthain; il publia dans leur langue, parmi d'autres titres, les auvres de Machiavel et de l'Arétin, les Annotationi sopra la Gierusalemme liberata de Scipione Gentili, la Descrittione del Regno di Scotia de Petruccio Ubaldini, une édition du Pastor fido, ainsi que plusieurs traductions. Sur la foi d'une indication donnée par De Thou, l'Aviso piacevole a 
généralement été attribué à François Perrot, un érudit parisien établi à Genève:

Ce placard [de Pierre de L'Estoile] fut suivi quelques temps après d'un écrit Italien beaucoup plus ample adressé à l'Italie, qui parut imprimé sous le nom d'un gentilhomme François. Cet ouvrage étoit farci de plusieurs pièces de poësies Italiennes composées contre le Pape et sa Bulle, par lesquelles on prétendoit lui donner le démenti. On y avoit cousu quelques morceaux tirés de Pétrarque, du Dante et de Boccace, où ces auteurs faisoient une satire très-piquante des vices et de la corruption de la Cour de Rome, avec cette liberté que leur siècle leur accordoit. On attribue cet ouvrage à François Perrot ${ }^{29}$.

De Thou connaissait bien Perrot, qui, comme lui, appartenait à la grande robe parisienne, et avec la famille duquel la sienne était alliée. Perrot était passé à la Réforme en suivant l'exemple de son oncle, le théologien Charles Perrot. Il avait longuement séjourné en Italie, à Padoue d'abord entre 1549 et 1555, puis à Venise, où il fut secrétaire de l'ambassadeur Arnaud du Ferrier, entre 1568 et 1572. Il acquit au cours de ces séjours, outre l'amitié de l'Arétin, une rare maîtrise de la langue italienne, au point de susciter l'admiration de De Thou qui précisait:

Il avoit depuis voyagé pendant longtemps en Italie, et à force de parler et d'écrire l'Italien, il en avoit si bien attrapé le goût que les naturels mêmes du pays y seroient trompés, et seroient obligés d'avouer qu'ils ne composent pas mieux en leur langue ${ }^{30}$.

Au contraire des autres "italianisants», Perrot ne traduisait pas d'ouvrages italiens en français, mais à l'inverse, de français en italien. On lui doit en particulier une version des Psaumes, ainsi que celle de La Vérité de la Religion Chrestienne de Du Plessis-Mornay, avec lequel il était très lié, publiée en 1584 à l'usage de la nation italienne de Genève. La rédaction des sonnets de l'Aviso piacevole répondait ainsi à un goût fort singulier pour la poésie toscane dont Perrot avait assimilé les formules, et à un engagement politique en faveur de Henri de Navarre.

L'attribution de l'Aviso piacevole dans son ensemble doit toutefois être précisée à la lumière de son lieu d'impression. Le volume publié en 1586 apparaît en effet comme un recueil, typographiquement homogène, mais composé de pièces de nature et d'origines distinctes, le tout réuni pour servir la cause de Henri de Navarre, probablement par l'entremise de Richard Hakluyt, le remuant secrétaire de l'ambassade d'Angleterre auprès de la cour de France. Il est constitué d'une strate "gallicane», la Risposta fatta 
alla scomunione di Sisto V, qui n'est autre que la traduction en italien du "placard" de Pierre de L'Estoile. Les 51 sonnets anti-romains, attribués à Perrot, constituent une autre strate, "protestante», italo-française. Le recueil souvre par un avis de l'imprimeur anonyme, en fait John Wolfe, et se poursuit par l'anthologie commentée des écrits hostiles à la papauté, tirés de Dante, de Boccace et de Pétrarque. Cette anthologie avait été réunie par les collaborateurs de Wolfe, et elle constitue une troisième strate, "protestante» certes, mais anglo-italienne. L'avis au lecteur peut être rapproché par son style des préfaces aux éditions de l'Arétin publiées chez Wolfe deux années plus tôt par le mystérieux Barbagrigia, que l'on a identifié avec le florentin Petruccio Ubaldini ${ }^{31}$. Or cette collection de textes anti-romains, avant sa mise au service de la propagande protestante, appartenait à une longue tradition florentine, codifiée dès la fin du XVé siècle. Un détail significatif permettra d'oter au calviniste Perrot l'attribution qui lui est habituellement faite du choix des textes et de leur commentaire. L'annotation qui accompagne l'extrait du chant XXXIII du Purgatorio identifie les "naiade" évoquées par Dante avec les grandes figures de la Réforme, Luther, Melanchton, les "Bâlois" Erasme, Bucer et Zwingli mais passe sous silence les Genevois Calvin et Théodore de Bèze. Or le cénacle italo-anglais qui réunissait les collaborateurs de Wolfe était en étroite relation avec le refuge italien de Bale, regroupé autour du libraire Perna ${ }^{32}$. Jacopo Castelvetro fut le go-between unissant Bâle et Londres, Perna et Wolfe : jouant le rôle d'un véritable directeur éditorial auprès de l'éditeur anglais, chez qui il publia un autre ouvrage contre l'excommunication des princes, il avait fait paraître chez le libraire lucquois de Bâle la grande édition "protestante» des ceuvres poétiques italiennes de Pétrarque, dont quelques exemplaires avaient été envoyés en France par l'intermédiaire de son collaborateur Betti ${ }^{33}$.

Les textes des "trois couronnes" et de Dante en particulier répondaient au dessein d'offrir un témoignage irrécusable de l'indignité des papes, fondé sur une autorité plus véridique qu'un écrit moderne et non italien. Ils devaient servir à prouver de façon éloquente que la décision de Sixte Quint était inique, non pas en termes étroitement politiques, mais religieux, parce que Rome était une nouvelle Babylone et le pape l'Antéchrist. Ils servaient enfin à révéler la vraie foi, capable de sauver la France, prise dans les guerres civiles que le pape attisait, mais aussi l'Italie, elle-même soumise à la tyrannie des successeurs de saint Pierre. Les citations du poème de Dante étaient complétées par des annotations tirées en partie du commentaire de Landino, qui traçaient un "naturale e vivo ritratto del Papa", dénonçant la cupidité, l'hérésie, la sodomie et la simonie des pontifes romains. 
Les exemples de Nicolas III et de Boniface VIII étaient les emblèmes de cette corruption. Les gloses ajoutées au commentaire actualisaient une vieille polémique, la situant au croisement de la littérature et de l'action politique. Deux passages en effet renvoyaient plus précisément à la situation française et à l'excommunication de Henri de Navarre, présentée comme la conséquence nécessaire d'une longue tradition d'arbitraire et de la prétention du Saint-Siège à l'hégémonie :

Carlo di Valois re di Puglia per haver negato al Papa la figliuola per suo nepote, fu scomunicato, et hebbe il papa ardimento di fargli la guerra ${ }^{34}$.

Et dans le passage où Béatrice prédit la ruine de la "meretrice romana" sous le signe d'un chef, d'un "dvx» que le commentateur Landino avait identifié avec Henri IV de Luxembourg, son glossateur dans l'Aviso piacevole préférait voir une prophétie annonçant le temps de la venue de Luther. Il précisait toutefois en offrant une solution plus conforme aux espoirs des protestants français :

Ma se pure d'un Arrigo duce o Duca si dee ciò a intendere, notiamo come hoggi ci sono due Arrici levati in piede (...) per le mani de quali dobbiamo sperare che questa prophetia si possa adempiere ${ }^{35}$.

Il nommait Henri de Condé et Henri de Navarre, appelés à détruire la prostituée romaine et le mauvais géant, "il re di Francia», avec qui elle commettait tous ses crimes. Un dernier passage enfin, tiré du chant XXXIII $d u$ Purgatorio, permettait au glossateur protestant de faire de Dante un adversaire de la messe et de donner un sens théologique à son interprétation politique.

La lecture anti-romaine des vers de Dante, abusive à bien des égards, connut une fortune toute particulière au cours de la seconde moitié du $X V I$ siècle. Avant d'ètre reconnu comme un poète, Dante était considéré comme un auteur politique, qui avait, en son temps, critiqué l'authenticité de la Donation de Constantin et qui avait affirmé l'autonomie politique de l'Empire. En 1559, le libraire bâlois Johann Oporinus publia, sous le titre De Monarchia, un recueil de textes d'auteurs italiens consacrés à la définition de l'imperium, parmi lesquels le traité de Dante ${ }^{36}$. Cette position strictement politique fut reprise par Papire Masson et par Pasquier pour appuyer la position gallicane des relations entre la royauté et l'Eglise, en forçant le guelfisme de Dante et en l'élargissant à l'ensemble des princes afin d'appuyer une autonomie politique que le poète toscan revendiquait 
pour l'Empereur. De leur côté, les protestants furent conduits à en infléchir le sens et à faire de Dante un adversaire de l'autorité pontificale, hostile à la papauté en tant que puissance spirituelle et non pas seulement politique. Contempteur des mours dissolues de la cour de Rome, il pouvait apparaître au prix de quelques ajustements et de quelques omissions comme un grand ancêtre de la Réforme, et sa vie d'exilé en faisait un très convenable martyr de la foi. Dès 1556, Matthias Flaccius Illyricus donnait, dans son Catalogus testium veritatis, également publié chez Oporinus, les clefs de cette interprétation quill étendait au poète :

Dantes Florentinus [...] scripsit librum quem appellavit Monarchiam. In eo provat Papam non esse supra Imperatores. Dicit alibi Papam esse ex pastore factum lupum [...] In cantione XXXIII Purgatorii non obscure ostendit Papam esse meretricem Babyloniam $^{37}$.

La formule de Flaccius Illyricus devint un "lieu» de la polémique protestante comme de la célébration du poète ou plutôt du penseur italien. En Angleterre, John Foxe, un des collaborateurs de l'édition bâloise du De Monarchia, la reprenait à la lettre dans son Book of Martyrs, en accentuant sa charge anti-romaine ${ }^{38}$. Les Français connurent le nom de Dante à travers cette médiation, et ils firent usage d'une formule désormais topique dans les controverses religieuses. Avant les événements de 1585, un des adversaires de Ronsard pouvait présenter le poète italien comme une victime de la papauté :
Depuis, Dante et Ocham, qui maintenoient l'Empire
Ne dépendre du Pape, à peine fuirent l'ire
Du douziesme Benoist qui de son Avignon
Le foudre à pleine gorge épandit sur leur nom ${ }^{39}$.

Après 1576 et la publication du Discours sur les moyens de bien gouverner de Gentillet, Dante servit de substitut à Machiavel, dont l'autorité appuyait jusqu'alors la dénonciation des vices de Rome. Il n'avait pas sur l'auteur du Prince et des Histoires florentines une préséance due à son antiquité ou à sa réputation savante. Mais Machiavel, découvert et diffusé en France par des érudits protestants dans les années 1540, par Milles II Perrot en particulier, l'oncle du "gentilhomme français" de l'Aviso piacevole, venait, à la suite d'une véritable manipulation idéologique, d'être désigné comme le responsable du "crime italien» de la Saint-Barthélémy et cessait d'être considéré autrement qu'en termes 
infamants ${ }^{40}$. Ce n'est pas un hasard si une génération plus tard, alors que sestompait la charge négative attribuée au nom même du secrétaire florentin, Machiavel et Dante pouvaient à nouveau être cités ensemble, en compagnie de Pétrarque, dans le Mystère d'Iniquité de Du PlessisMornay, utilisés tous les trois dans un même discours anti-romain qui retrouvait à propos de Dante les termes même de la formule du Catalogus de Flaccius Illyricus :

Florissoit de ce temps Dante Florentin, recommandé par les escrivains du temps de piété et de doctrine. Il fit un traité intitulé Monarchia où il prouve que le Pape n’est point au-dessus de l'Empereur, et n'a aucun droit sur l'Empire ${ }^{41}$.

En en alléguant en marge le chant XXXIII du Purgatorio ainsi que les chants IX et XXIX du Paradiso, Du Plessis-Mornay rappelait que :

Plusieurs autres lieux s'en pourroient tirer contre les pardons et indulgences du pape, et autres abus de l'Eglise romaine qu'il nous depeint de sorte qu'il est aisé de voir qu'il avoit bien remarqué la Paillarde de l’Apocalypse ${ }^{42}$.

L'Aviso piacevole était une des nombreuses variations sur ce "lieu» rhétorique de la pensée protestante. Ce livre étrange, composé d'une matière italienne, édité en Angleterre, opportunément publié pour servir l'action de Henri de Navarre, était destiné à des lecteurs italiens ou italianisants de Genève, de Bâle, de Londres ou de Paris plus quiaux Italiens de Florence ou de Rome qu'il ne pouvait guère toucher. Le nonce Ragazzoni, dans deux lettres adressées au cardinal Rusticucci, évoquait les autres livres suscités par le bref de Sixte Quint, le Brutum Fulmen de Hotman et le traité de Pierre de Belloy, dont il désignait le commanditaire, le garde des Sceaux Cheverny ${ }^{43}$. Ces deux ouvrages, que le nonce avait eu beaucoup de peine à se procurer, furent immédiatement mis à l'Index. L'Aviso piacevole au contraire semble être passé presque inaperçu. Il ne put échapper toutefois à la curiosité de Jacques Cappel, dont on conserve encore l'exemplaire ${ }^{44}$, et à la vigilance de Robert Bellarmin, venu en France avec la légation du cardinal Caetani. Bellarmin citait le recueil dans son appendix au traité De summo Pontefice publié dans le De Controversiis christianæ fidei, et il entreprit de le réfuter ${ }^{45}$. Bellarmin réhabilitait sinon le poète du moins le penseur, dont il confirmait l'orthodoxie, mais soulignait l'incompétence en matière de dogme. Soucieux toutefois de ne pas laisser un tel auteur dans les mains des protestants, il reprenait point par point les extraits cités dans l'Aviso piacevole, pour les réfuter à l'aide du commentaire même de 
Landino et faire des critiques de la Rome pontificale une critique catholique de la Rome païenne ${ }^{46}$.

Le pamphlet enfin fut lu par le plus attentif des lecteurs et le meilleur connaisseur de Dante, non pas un Français, ni un protestant, mais Corbinelli, l'éditeur du De Vulgari Eloquentia. Les notes qu'il laissa sur son exemplaire portent, à trois exceptions près, sur les vers de la Commedia. C'étaient des gloses philologiques, comme si leur auteur, érudit solitaire dans une ville tout agitée des manœuvres de la Ligue, était indifférent à l'intention polémique qui avait présidé à la composition du recueil. Corbinelli ne tirait à son tour du texte quil avait sous les yeux que l'occasion d'une nouvelle confrontation entre le toscan ancien dont il avait ailleurs récusé l'exemplarité et l'italien moderne dont il cherchait la solution ${ }^{47}$.

Jean Balsamo 
1. La Comédie de Dante, De l'Enfer, du Purgatoire et Paradis, Paris, J. Gesselin, G. Drobet et la veuve G. Drobet, 1596-1597; exemplaire de dédicace à Henri IV conservé à la B.N.F. (Rés. Yd 817-819). Sur le traducteur, voir L. Dorez, Balthazar Grangier, dans Mélanges sur Dante, éd. par M. Mignon, Rome, 1931, p. 426-429. Fils de Jean Grangier de Liverdis, ambassadeur auprès des Grisons, Balthazar Grangier était chanoine de N.D. de Paris et abbé de Saint-Barthélémy de Noyon depuis 1573. Traducteur de Julien l'Apostat, il avait collaboré en 1583 au Tumulus de René de Birague. Il mourut en 1606.

2. A. Farinelli, Dante e la Francia dall'età media al secolo di Voltaire, Milan, 1908.

3. F. Simone, Dante e la cultura francese del Rinascimento, dans Umanesimo, Rinascimento, Barocco in Francia, Milan, 1968, p. 150-167 ; voir également D. Cecchetti, Dante e il Rinascimento francese, Letture classensi, 19 (1990), p. 35-63.

4. Etienne Pasquier dénonça la "sotte opinion de Dante Poëte Italien» qui qualifiait Hugues Capet de fils de boucher, et il diffusa la légende d'un François Ier indigné à la lecture de la Commedia par Luigi Alamanni, Les Recherches de la France, Amsterdam, 1723, t. I, col. 513-514.

5. Sur ces éditions, voir E. Picot, Les Français italianisants au XVIe siècle, Paris, 1906, t. I, pp. 171172 et 183-220 ; N. Bingen, Le Mâttre italien (1510-1660), Bruxelles, 1987, p. 267-269.

6. Voir M. Connat, Mort et testament de Remi Belleau, B.H.R., 6 (1945), p. 328-356.

7. Pierre de L'Estoile, Mémoires-Journaux, éd. par Brunet, Champollion, Tamisey de Larroque, Halphen, Paris, 1875-1896, t. IX, p. 173.

8. H. Jadart, Inventaire du mobilier et de la bibliothèque de Nicolas Colin, Arcis sur Aube, 1892 , p. 55.

9. A. Vidier, Un bibliophile du XVIe siècle, Nicolas Moreau d'Auteuil, dans Mélanges offerts à E. Picot, Paris, 1913, t. II, p. 371-377.

10. Cet exemplaire est conservé à la B.N.F. (Rés. Yd 807).

11. Bibliothèque H. Destailleur, 1891, n 1170.

12. Inferno, XI, 93; Essais, I, 26, Edition municipale, Bordeaux, 1906, t. I, p. 196 ; Purgatorio, XXVI, 34-36 ; Essais, II, 12, éd. cit., t. II, p. 167.

13. Voir P. Ronzy, Un Humaniste italianisant, Papire Masson (1544-1611), Paris, 1924, p. 367 et 405-407.

14. Antoine Du Verdier, La Prosopographie, Lyon, 1573, p. 50, 301, 339 et 436.

15. Jean de La Gessée, Les premières Oeuvres françoyses, Anvers, Plantin, 1583, t. II, p. 664-665; Gui Le Fèvre de La Boderie, Hymne à la Mère de Dieu de Dante poëte Toscan, dans Hymnes ecclésiastiques selon le cours de l'année, Paris, R. Le Mangnier, 1578, f. 167-169; sur la traduction de Le Fèvre, déjà citée par Farinelli, p. 552, voir R. Gorris, Alla Corte del Principe, Ferrare, 1996, p. 91.

16. Sur ce discours polémique des érudits français, nous nous permettons de renvoyer à notre étude Les Rencontres des Muses. Italianisme et anti-italianisme en France au XVIe siècle, Genève, 1992, p. $177-216$ 
17. Voir notre Note sur l' Elégie à Bartolommeo Delbene Florentin», dans Revue des Amis de Ronsard, 10 (1997), p. 145-162. La référence à Dante et Cavalcanti provenait du commentaire de Muret aux Amours, sonnet XIV.

18. Gui Le Fèvre de La Boderie, La Galliade ou la révolution des arts et des sciences, Paris, G. Chaudière, 1578, f. 122-123.

19. P. Simoncelli, La Lingua di Adamo: Guillaume Postel tra Accademici e Fuorusciti Fiorentini, Florence, 1984, p. 115-164 ; voir, dans une même perspective, P. Cherchi, Corbinelli, Postel e il problema dell'antico toscano, dans Actes du colloque Guillaume Postel, Paris, 1984, p. 317-326.

20. Voir M. Fumaroli, "Aulae arcana". Rhétorique et politique à la cour de France sous Henri III et Henri IV, dans Journal des Savants, 1981, p. 137-189; Corbinelli avait corrigé un exemplaire pour une seconde édition qui ne fut pas publiée, voir C. S. Gutkind, Die handschriftlichen Glossen des Iacopo Corbinelli zu seiner Ausgabe der "De Vulgari Eloquentia" Paris 1577, dans Archivum romanicum, 17 (1934), p. 19-120 ; sur le "misogallisme" linguistique de Dante, voir P. V. Megaldo, Linguistica e retorica in Dante, Pise, 1978, p. 294-303.

21. Vitae trium Hetruriae Procerum, Paris, Du Pré, 1587 , f. $3 \mathrm{v}^{\circ}$. L'exemplaire de Tamisier est conservé à la Bibliothèque municipale de Troyes (T. 13.1636) ; Tamisier fit paraître sa traduction de la Méditation sur le "Miserere mei» de Savonarole dans ses Méditations chrestiennes, Paris, A. L'Angelier, 1588.

22. Claude Duret, Thrésor des langues de cest Univers, Yverdon, 1619, p. 807.

23. Voir P. Ronzy, Dante auxiliaire du gallicanisme, dans Dante. Mélanges de critique et d'érudition, Paris, 1921, p. 126-135.

24. René de Lucinge, Lettres sur le début de la Ligue, éd. A. Dufour, Genève, 1964, p. 209.

25. Jacques-Auguste de Thou, Histoire, Londres, 1734, t. IX, p. 376.

26. François Hotman, Protestation et defense pour le roy de Navarre. Traduicte du latin intitulé Brutum Fulmen, [s.1.], 1587, p. 234.

27. Aviso piacevole dato alla bella Italia da un nobile giovane Francese, sopra la mentita, data dal Serenissimo Re di Navarra a Papa Sisto V, "Monaco, appresso Giovanni Swartz" [Londres, John Wolfe], 1586, f. 13-27 $\mathrm{v}^{\circ}$ : exemplaires consultés : Paris, BNF (K. 2017 et Rés. H. 989), Arsenal ( $4^{\circ}$ H 2884), Sainte-Geneviève ( $4^{\circ} 549$ inv. 375 Rés.).

28. Voir D.B. Woodfield, Surreptitious Printing in England (1550-1640), New York, 1973, p. 5, $\mathrm{n}^{\circ} 11$ et p. $109, \mathrm{n}^{\circ} 42$.

29. De Thou, Histoire cit., p. 378 ; sur Perrot, voir E. Picot, Les Français italianisants, Paris, 1906, t. I, p. 324-380.

30. De Thou, Histoire cit., p. 378.

31. Voir M.G. Bellorini, Le pubblicazioni italiane dell'editore Londinese John Wolfe (1580-1591), dans Pubblicazioni della Facoltà di lingue dell'Università di Trieste, sede di Udine, 1971, p. 17-65.

32. Voir P. Ottolenghi, Giacopo Castelvetro esule modenese nell'Inghilterra di Shakespeare, Pise, 1982. 
33. Voir L. Perini, Note e documenti su P. Perna libraio-tipografo a Basilea, dans Nuova Rivista Storica, 50 (1966), p. 155.

34. Aviso piacevole, f. 15.

35. Aviso piacevole, f. $21 v^{\circ}$

36. Voir L. Sebastio, Capitoli sulla "Monarchia" di Dante nel Protestantesimo (1550-1560), dans Annali della Facoltà di Lettere e Filosofia di Bari, 15 (1972), p. 338-384.

37. Cité d'après Sebastio, Capitoli cit., p. 344.

38. Voir John Tedeschi, L'oeuvre littéraire de la Réforme italienne, dans Actes du colloque "Le Livre à la Renaissance», Tours, 1985, Paris, 1988, p. 406, ainsi que Ch. Dédayan, Dante en Angleterre, Paris, 1961, p. 121-125.

39. Remonstrance à la Royne sur le discours de Pierre de Ronsard [Lyon, Le Clerc, 1563], dans La Polémique protestante contre Ronsard, éd. J. Pineaux, Paris, Société des Textes français modernes, 1973, p. 148. Pasquier reprit la formule dans ses Recherches de la France, Amsterdam, 1723, t. I, col. 217.

40. Voir notre étude "Un livre écrit du doigt de Satan". La découverte de Machiavel et l'invention du Machiavélisme en France au XVI siècles, dans Le Pouvoir des livres à la Renaissance, éd. par D. de Courcelles, Paris, 1998, p. 77-92.

41. Philippe Du Plessis-Mornay, Le Mystère d'Iniquité c'est à dire l'histoire de la Papauté, Saumur, Portau, 1611, p. 419-420.

42. Du Plessis-Mornay, Le Mystère cit., p. 420.

43. Girolamo Ragazzoni, Correspondance de sa nonciature (1583-1586), éd. par P. Blet, RomeParis, 1962, lettres du 25 mai et du 23 juin 1586.

44. Ex-libris daté 1597, exemplaire conservé à la bibliothèque de l'Arsenal (4 H 2884); Jacques Cappel est le neveu de Guillaume Cappel, traducteur, en 1553, du Prince de Machiavel.

45. R. Bellarmin, Disputationes de controversiis christianae fidei, Paris, 1613, t. I., col. 1013-1046.

46. Voir R. Ronzy, Bellarmin et Dante, dans Mélanges sur Dante, cit., p. 92-108, ainsi que P. Friedrich, Dante's Fame abroad, Rome, 1950, p. 81-83.

47. L'exemplaire de Corbinelli est conservé à la B.N.F. (Rés. H. 989). La Biblioteca Trivulziana de Milan possède un exemplaire du Discorso sopra la prima cantica del divinissimo Dante de Vincenzo Buonanni (Florence, 1572), également annoté par Corbinelli, dont les notes n’ont pas encore fait l'objet d'une étude particulière. 